The final publication is available at Elsevier via https://doi.org/10.1016/j.jcsr.2019.105811. @ <2019>. This manuscript version is made available under the CC-BY-NC-ND 4.0 license http://creativecommons.org/licenses/by-nc-nd/4.0/

\title{
Shear Deformation Effects on Stability of Unbraced Steel Frames in Variable Loading
}

\author{
Terence $\mathrm{Ma}^{1}$, Lei $\mathrm{Xu}^{1}$ \\ ${ }^{1}$ Department of Civil and Environmental Engineering, University of Waterloo, 200 University \\ Avenue West, Waterloo, ON., N2L 3G1, Canada \\ Corresponding Author: \\ Lei Xu, University of Waterloo \\ 200 University Avenue West \\ Waterloo, ON. N2L 3G1 \\ Tel: (519) 888-4567 x36882 \\ Email: 1xu@uwaterloo.ca
}

\begin{abstract}
The generalized lateral stiffness equation of a semi-rigidly connected frame with accounting for shear deformation is derived in this paper. The lateral stiffness equation can be used to determine the critical loads of frames, as instability occurs when the lateral stiffness diminishes to zero. The proposed method is numerically exact, and is obtained via solving the Timoshenko system of governing differential equations for a semi-rigidly connected column. It is demonstrated in the derivation that the effect of shear deformation can conveniently be accounted for via use of the shear flexibility coefficient, $\eta$. The proposed equations are validated via finite element analysis and demonstrated via numerical examples. The concept of variable loading originally proposed by $\mathrm{Xu}$ is also generalized to include the effect of shear deformation, whereby the worst and best case distributions of axial loads in frames can be determined via solving a minimization problem. Although the effect of shear deformation can generally be neglected in practice regardless of whether proportional or variable loading are considered, this study demonstrates that the effect of shear deformation on the lateral stiffness and critical loads can be significant and detrimental if the frames contain supporting columns with low slenderness ratios and/or lean-on columns. From the results of the numerical examples, the critical load of a lean-on frame containing a lowslenderness supporting column $(L / r<40)$ can be reduced by up to $41 \%$ even when the supporting column is not loaded.
\end{abstract}

Keywords: shear deformation; unbraced steel frame; lateral stability; variable loading; Timoshenko; semi-rigid 


\section{$1 \quad$ Introduction}

The prevention of instability in the design of structural frames is of primary importance, as the instability of a structure can have catastrophic consequences to the life and safety of its occupants. As such, it is important for designers to have access to accurate models with regards to the stability analysis of structures. The concept of storey-based stability was initiated by Yura [1] for the assessment of lateral stability in an entire structural frame contained within a storey of a building by considering the interactions between the members in the frame. Various methods for evaluating the storey-based stability of steel frames have subsequently been proposed [1-5]. Although the effect of shear deformation can generally be neglected in practice, as in the case of these methods, the effect of the shear deformation on storey-based stability remains to be investigated as it is known to be detrimental to the critical loads in certain cases, such as when the columns have low slenderness ratios [6]. For instance, studies [7-8] have shown that shear deformations can have significant effects on the critical loads in built-up columns, which tend to have low slenderness ratios. However, structural members are conventionally analyzed with adopting the Euler-Bernoulli model of deformation [9], which includes an assumption that plane sections remain plane [10], and in doing so, neglects the effects of shear deformations. This assumption is not accurate in members with low slenderness ratios. As such, the Euler-Bernoulli model can be replaced with the Timoshenko model [11], which replaces the governing differential equation relating to the deformation of a member with a more accurate system of governing differential equations which distinguishes the slope of the centerline of a member from the slope of the normal to the cross-section. The Timoshenko [11] model has been validated experimentally [12-13] and is widely accepted in modern analysis procedures relating to the calculation of deformations with consideration for shear deformations [14-17]. The shear 
flexibility coefficient [6-7], which is an indicator for the relative influence of shear deformations on the critical loads of columns, depends largely on the slenderness ratio. Owing to the common use of steel members with low slenderness ratios in steel frames, the influence of shear deformations should be considered in storey-based stability analysis.

\section{$2 \quad$ Background}

It is well known that the interactions among different columns in a storey of an unbraced frame should be considered in the evaluation of stability in structural systems. The storey-based stability approach was initiated by [1] and has been developed over the last few decades to evaluate the lateral stability of steel frames. $\mathrm{Xu}$ [5] extended this approach by deriving the lateral stiffness equation with consideration of the effect of the column axial load for a planar storey frame with $n$ bays shown in Fig. (1) and containing semi-rigid connections.

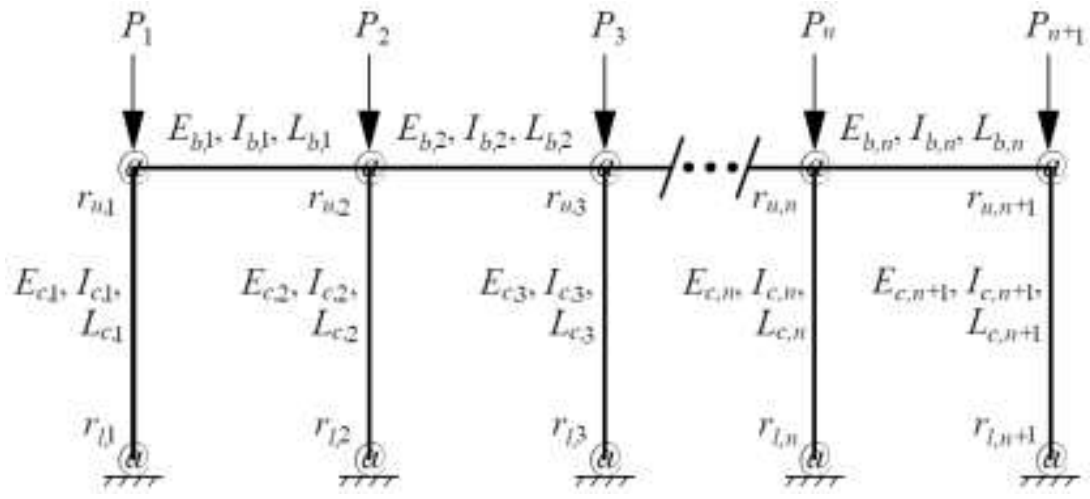

\section{Figure 1 - General Unbraced Storey Frame subjected to Gravity Loading}

The planar frame is subjected to applied gravity loads, $P_{i}$. Let the indices $i$ and $j$ correspond to the numbering of the columns and beams, respectively. Similarly, the subscripts $c$ and $b$ correspond to columns and beams, respectively. The elastic modulus, moment of inertia and length of each member are $E$, $I$, and $L$, respectively. All connections are generalized as rotational springs, and the column lower and upper end fixity factors are $r_{l}$ and $r_{u}$, respectively. The end 
fixity factors are measures of the rotational rigidity at corresponding ends of the member, originally defined by Monforton and Wu [19] and shown in Eqs. (1):

$$
\begin{gathered}
r_{u, i}=\frac{1}{1+3 E_{c, i} I_{c, i} / R_{u, i} L_{c, i}} ; \quad R_{u, i}=\sum_{j_{u}=1}^{m_{u}} R_{i, j_{u}} \\
r_{l, i}=\frac{1}{1+3 E_{c, i} I_{c, i} / R_{l, i} L_{c, i}} ; \quad R_{u, i}=\sum_{j_{l}=1}^{m_{l}} R_{i, j_{l}}
\end{gathered}
$$

where $R_{u}$ and $R_{l}$ are the rotational stiffness of the upper and lower end connections of the column, respectively. The end fixity factors are defined such at $r=0$ represents a pinned connection, and $r=1$ represents a fixed connection. Intermediate values of $r$ between zero and unity can be used to represent semi-rigid connections. The rotational restraint provided by beam $j$ to column $i$ at the corresponding end, $R_{i, j}$, can be calculated using Eq. (2) as follows [5].

$$
R_{i, j}=\frac{6 E_{b, j} I_{b, j} r_{N, j}}{L_{b, j}}\left[\frac{2+v_{j} r_{F, j}}{4-r_{N, j} r_{F, j}}\right]
$$

where $r_{N, j}$ and $r_{F, j}$ are the end-fixity factors for the near and far ends of beam $j$ connected to column $i$, and $v$ is the ratio of rotation of the far-end connection of the beam, $\theta_{F}$, to the rotation of the near-end connection of the beam, $\theta_{N}$. Xu and Liu [20] demonstrated that accurate estimations of results can be obtained by assuming the case of asymmetric buckling, $v=1$, for unbraced partially restrained frames. Xu [5] showed that the lateral stiffness of the frame in Fig. (1), with ignoring the effect of shear deformation, can be calculated using Eq. (3).

$$
\Sigma S=\sum_{i=1}^{n+1} S_{i}=\sum_{i=1}^{n+1} \frac{12 E_{c, i} I_{c, i}}{L_{c, i}^{3}} \beta_{i}\left(\phi_{i}, r_{l, i} r_{u, i}\right)
$$

Where $\Sigma S$ is the lateral stiffness of the storey frame, taken as the sum of lateral stiffness of each column, $S_{i}$. The $\beta_{i}$ factor accounts for second-order effects, shown in Eqs. (4).

$$
\begin{gathered}
\beta=\frac{\phi^{3}}{12} \frac{a_{1} \phi \cos \phi+a_{2} \sin \phi}{18 r_{l} r_{u}-a_{3} \cos \phi+\left(a_{1}-a_{2}\right) \phi \sin \phi} \\
a_{1}=3\left[r_{l}\left(1-r_{u}\right)+r_{u}\left(1-r_{l}\right)\right]
\end{gathered}
$$




$$
\begin{gathered}
a_{2}=9 r_{l} r_{u}-\left(1-r_{l}\right)\left(1-r_{u}\right) \phi^{2} \\
a_{3}=18 r_{l} r_{u}+a_{1} \phi^{2}
\end{gathered}
$$

Where $\phi_{i}=L_{i} \sqrt{P_{i} / E_{i} I_{i}}$ is the axial load coefficient. $\beta_{i}$ is a monotonically decreasing function of the axial load, and indicates whether the column is sufficiently stable on its own $(\beta>0)$ or relies on the lateral stiffness of other columns in the storey to maintain stability $(\beta \leq 0)$. The frame is laterally stable if $\Sigma S>0$, and unstable when $\Sigma S$ diminishes to zero since theoretically the lateral deformations would approach infinity. Note that Eq. (3) can conveniently be extended towards semi-braced frames by adding the lateral stiffness of the bracing system to $\Sigma S$ [21]. Xu [5] also introduced the concept of variable loading, which determines the worst and best cases of applied loads that can result in the instability of an unbraced frame. The worst and best case scenarios are obtained by solving the minimization and maximization problems shown in Eqs. (5), respectively, for an $n$-bay frame [5].

$$
\min \text { or } \max : \sum_{i=1}^{n+1} P_{i}
$$

subject to:

$$
\begin{gathered}
\Sigma S=\sum_{i=1}^{n+1} S_{i}=\sum_{i=1}^{n+1} \frac{12 E_{c, i} I_{c, i}}{L_{c, i}^{3}} \beta\left(\phi_{i}, r_{l, i}, r_{u, i}\right)=0 \\
0 \leq P_{i} \leq P_{u, i}=\frac{\pi^{2} E_{c, i} I_{c, i}}{\left(K_{i} L_{c, i}\right)^{2}}
\end{gathered}
$$

By minimizing the total axial load and setting the instability of the frame as a constraint in Eq. (5b), the least amount of loading causing instability can be determined. Similarly, by maximizing the total axial load the most amount of loading that can be applied before instability occurs can be determined. The constraint in Eq. (5c) is required as columns cannot exceed their rotational buckling loads, $P_{u, i}$, and are assumed to be under compression. $K_{i}$ is the effective length of the column. The minimization problem can readily be solved using mathematical programming. 
The lateral stiffness equation in Eq. (3) was derived using the Euler-Bernoulli assumption, which ignores the reduction in lateral stiffness resulting from shear deformation by assuming that plane sections remain plane. This assumption is valid for sufficiently slender members where the shear flexibility factor, $\eta$, is sufficiently small in Eq. (6) [6].

$$
\eta=\frac{E I / L^{2}}{\kappa A G}=\frac{E}{G \kappa}\left[\frac{1}{(L / r)^{2}}\right]
$$

Where $r$ is the radius of gyration, $\kappa A$ is the effective shear area and $G$ is the shear modulus given in Eq. (7).

$$
G=\frac{E}{2(1+v)}
$$

where $v=0.3$ is the Poisson's ratio of steel at ambient temperature. However, there are many cases where the members are not sufficiently slender. As such, the reduction in the lateral stiffness due to shear deformation would significantly reduce the obtained critical loads of the frame. Moreover, the consideration of shear-deformation effects can only reduce the lateral stiffness of members. As such, the Xu [5] method could yield non-conservative results of the critical loads in some cases. To address this shortcoming, the lateral stiffness equation with considering shear deformation is derived in this paper via use of the Timoshenko beam assumption [11]. Unlike Euler-Bernoulli members, plane sections are not assumed to remain plane in Timoshenko beams, as shown in Fig. (2), in which $\varphi$ is the rotation of the normal of the section to the horizontal axis. Unlike Euler-Bernoulli members where plane sections remain plane, $\varphi$ is not equal to the derivative of $y$ with respect to $x$. Throughout this paper, positive internal moments, $M(x)$, are assumed to induce compression in the bottom-side fibers of the column, and positive internal shears, $V(x)$, are assumed to cause counter-clockwise rotation of the member, as illustrated in the directions shown in Fig. (2). 


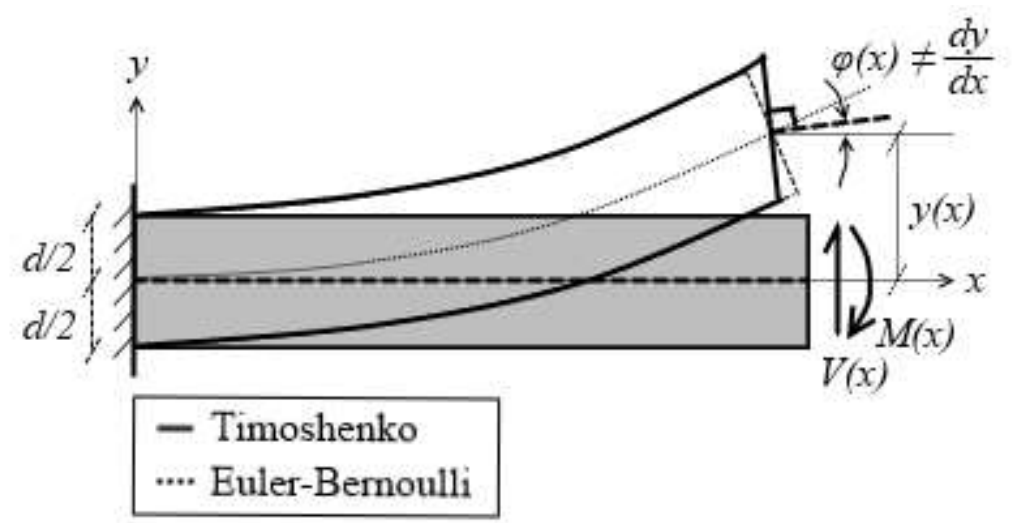

Figure 2 - Visual comparison of Timoshenko versus Euler-Bernoulli members

Note that in Fig. (2), $M(x)$ is drawn positive-clockwise as consistent with the sign convention used in this paper, but the beam is shown to deform as if $M(x)$ is negative. Also, $V(x)$ has been considered by Engesser [22] to act perpendicular to the normal to the section and by Haringx [23] to act perpendicular to the normal to the slope of the member. As there is considerable disagreement in the literature over which is more accurate [6,24], solutions with accommodating both of these assumptions are presented and compared in this study. The deformation of a Timoshenko beam is governed by Eq. (8) [11].

$$
E I \frac{d^{4} y}{d x^{4}}=q(x)-\frac{E I}{\kappa A G} \frac{d^{2} q}{d x^{2}}
$$

where $A$ is the cross-sectional area. $y(x)$ is the transverse deflection of the member at location $x$ along the length of the member. $q(x)$ is the transverse loading function. $\kappa$ is the Timoshenko shear coefficient of the section based on its geometry. Theoretically $\kappa$ is the value satisfying Eq. (9) and can be approximated using equations from Cowper [25] for various shapes.

$$
\int_{A} \tau d A=\kappa A G \varphi
$$


For Euler-Bernoulli beams, the last term in Eq. (8) is omitted and is valid when $\eta$ is very small. Based on Eq. (9), the moment and shear functions, $M(x)$ and $V(x)$, respectively, are given in Eqs. (10).

$$
\begin{gathered}
M(x)=-E I \frac{d \varphi}{d x} \\
V(x)=\kappa A G\left(-\varphi(x)+\frac{d y}{d x}\right)
\end{gathered}
$$

\section{Storey-Based Stability with Considering the Effects of Shear Deformation}

The lateral stiffness of an unbraced frame containing semi-rigid connections and with considering the effect of shear deformation via Timoshenko columns [11] is derived in this section. The proposed method is a generalization of the method presented in [5] for computing the lateral stiffness of a semi-rigidly connected frame developed from the theory of EulerBernoulli columns, which neglects shear deformation. The effect of shear deformation on the lateral stiffness of columns is conveniently accommodated via the introduction of a shear influence factor. To be consistent with the method presented in [5], the proposed method also accounts for the reduction in lateral stiffness due to axial loads via $P-\Delta$ effects. It is further noted that the proposed equations are explicit closed-form solutions to the governing differential equations, which can conveniently be computed in spreadsheets and are computationally efficient compared to other methods such as eigenvalue buckling analysis as it does not require the construction of a stiffness matrix nor the solution to an eigenvalue problem.

\subsection{Lateral Stiffness of a Semi-Rigid Column}

First, the semi-rigidly connected column in Fig. (3) is considered. 


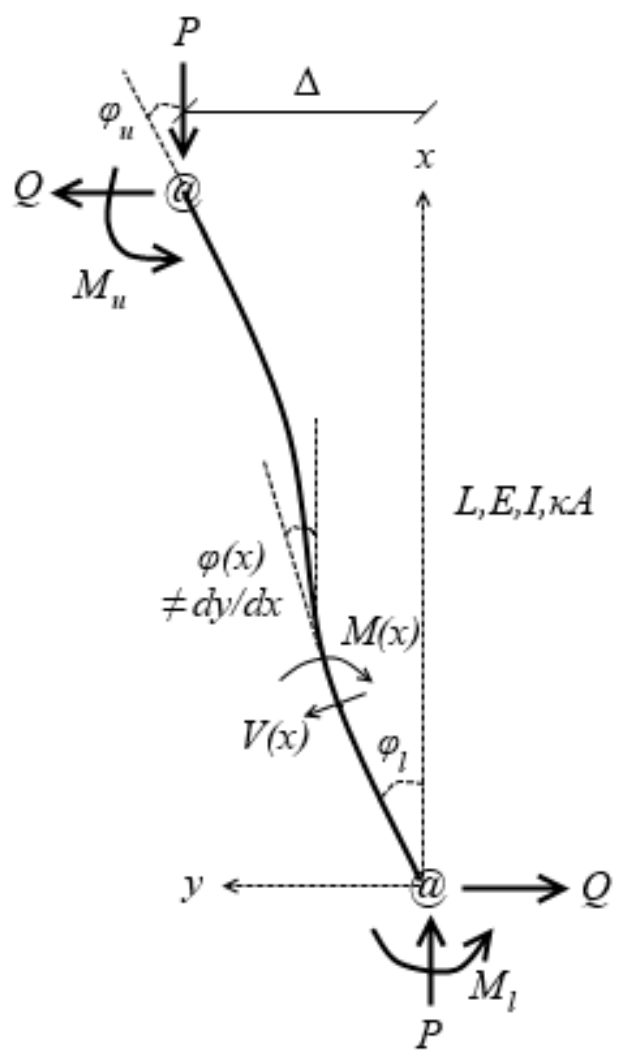

\section{Figure 3 - Semi-rigidly connected Timoshenko column}

The deformation of the column is assumed to be based on the Timoshenko beam equations in Eqs. (10). The end moments $M_{u}$ and $M_{l}$ relate to the additional end rotations, $\varphi_{l}$ and $\varphi_{u}$, in Eq. (11).

$$
\begin{gathered}
M_{u}=R_{u} \varphi_{u} \\
M_{l}=R_{l} \varphi_{l}
\end{gathered}
$$

The end moments are shown in the positive counter-clockwise convention but act in the opposite direction. As such, in assuming small deformations the moment and shear functions of the column can be expressed in Eq. (12).

$$
\begin{gathered}
M(x)=-R_{l} \varphi_{l}+Q x+P y=-E I \frac{d \varphi}{d x} \\
V(x)=Q+P \varphi=\kappa A G\left(-\varphi(x)+\frac{d y}{d x}\right)
\end{gathered}
$$


Note that in Eq. (12b), the shear function $V(x)$ may alternatively be taken as $Q+P y^{\prime}$, where $y^{\prime}$ is the derivative of $y(x)$ with respect to $x$. Eq. (12b) corresponds to the assumption of Engesser [22] whereas $V(x)=Q+P y^{\prime}$ corresponds to the assumption of Haringx [23]. In terms of the applications in this study it will be demonstrated that both assumptions yield virtually identical results. It is noted that Timoshenko himself [26] adopted the assumption of Haringx [23]. Regardless of which assumption is used, taking moments about the base of the column thus gives the following relationship between the end rotations in Eq. (13).

$$
\varphi_{l} R_{l}+\varphi_{u} R_{u}=Q L+P \Delta
$$

Solving the system of differential equations in Eqs. (12) results in the deformed shape and rotation of the column expressed in Eqs. (14).

$$
\begin{gathered}
y(x)=C_{1} \cos \left(\frac{\phi \omega}{L} x\right)+C_{2} \sin \left(\frac{\phi \omega}{L} x\right)+\frac{\varphi_{l} R_{l}}{P}-\frac{Q}{P} x \\
\varphi(x)=\frac{1}{\omega^{2}}\left[C_{2} \frac{\phi \omega}{L} \cos \left(\frac{\phi \omega}{L} x\right)-C_{1} \frac{\phi \omega}{L} \sin \left(\frac{\phi \omega}{L} x\right)\right]-\frac{Q}{P}
\end{gathered}
$$

where $\phi$ is the axial load coefficient, $\omega$ is the axial shear modifier coefficient given in Eqs. (15), and $C_{1}$ and $C_{2}$ are integration constants that depend on the boundary conditions. By the assumption of Engesser [22] $\omega$ will take the form of Eq. (15a), while $\omega$ will take the form of Eq. (15b) by the assumption of Haringx [23].

$$
\begin{gathered}
\omega=\sqrt{1+\frac{P}{\kappa A G}}=\sqrt{1+\eta \phi^{2}} \\
\omega=\frac{1}{\sqrt{1-\frac{P}{\kappa A G}}}=\frac{1}{\sqrt{1-\eta \phi^{2}}}
\end{gathered}
$$

Eqs. (15) reinforce the fact that the influence of shear deformation is related to $\eta$ [6]. Eq. (15b) is valid for $P<\kappa A G$, and asymptotically approaches infinity as $P$ approaches $\kappa A G$. As such, values above this limit are invalid. Similarly, for structural applications, $\eta$ is on the order of $10^{-3}$ to $10^{-4}$ 
and has negligible influence on the value of $\omega$. There are four boundary conditions to this problem, listed in Eqs. (16).

$$
\begin{gathered}
y(0)=0 \\
y(L)=\Delta \\
\varphi(0)=\varphi_{l} \\
\varphi^{\prime}(L)=\varphi_{u}
\end{gathered}
$$

Solving the system of five equations comprising of Eq. (13) and Eqs. (16) and isolating for the term $Q / \Delta$ results in the lateral stiffness, $S$, for the column, given in Eq. (17).

$$
S=\frac{Q}{\Delta}=\left[\frac{12 E I}{L^{3}} \beta^{\prime}\right]\left(\frac{1}{1+\zeta^{\prime}}\right)
$$

Where $\beta$ ' is a modified form of Eq. (4) given in Eq. (18a) and $\zeta^{\prime}$ is given in Eq. (18b).

$$
\begin{gathered}
\beta^{\prime}=\frac{\phi^{3} \omega}{12} \frac{a_{1}{ }^{\prime} \phi^{\prime} \cos \phi^{\prime}+a_{2}{ }^{\prime} \sin \phi^{\prime}}{18 r_{l} r_{u}-a_{3}{ }^{\prime} \cos \phi^{\prime}+\left(a_{1}{ }^{\prime}-a_{2}{ }^{\prime}\right) \phi^{\prime} \sin \phi^{\prime}} \\
\zeta^{\prime}=\left(\omega^{2}-1\right)\left[\frac{18 r_{l} r_{u}\left(1-\cos \phi^{\prime}\right)+a_{1}{ }^{\prime} \phi^{\prime} \sin \phi^{\prime}}{18 r_{l} r_{u}-a_{3}{ }^{\prime} \cos \phi^{\prime}+\left(a_{1}{ }^{\prime}-a_{2}{ }^{\prime}\right) \phi^{\prime} \sin \phi^{\prime}}\right] \\
a_{1}{ }^{\prime}=3\left[r_{l}\left(1-r_{u}\right)+r_{u}\left(1-r_{l}\right)\right] \\
a_{2}{ }^{\prime}=9 r_{l} r_{u}-\left(1-r_{l}\right)\left(1-r_{u}\right)\left(\phi^{\prime}\right)^{2} \\
a_{3}{ }^{\prime}=18 r_{l} r_{u}+a_{1}\left(\phi^{\prime}\right)^{2} \\
\phi^{\prime}=\phi \omega
\end{gathered}
$$

Note that in Eqs. (18), $\omega$ is given as either Eq. (15a) or Eq. (15b) depending on whether the assumptions of Engesser [22] or Haringx [23] are used, respectively. As consistent with the Euler-Bernoulli assumption, when $\eta$ is small, $\zeta^{\prime}$ is small and can be neglected, and Eq. (16) converges to Eq. (3). Of course, this also occurs for a shear-rigid member $(G=\infty, \eta=0)$. It can be shown that the consideration of shear deformation always reduces the lateral stiffness of a column in compression compared to when shear deformations are neglected. Thus, the effect of shear deformation on the lateral stiffness is accounted for solely via the shear flexibility coefficient $\eta$ in Eq. (18), and increases with the axial load, as predicted by Wang et al. [6]. Eqs. (18) is a generalized form of the lateral stiffness equation of a semi-rigid column, to which the 
derivation in [5] is a special case where $\eta=0$. Note that in the absence of applied gravity loads, $\zeta$ ' converges to the limit in Eq. (19a), and $\beta$ ' converges to Eq. (19b).

$$
\begin{gathered}
\lim _{\phi \rightarrow 0^{+}} \zeta^{\prime}=\frac{12 E I}{L^{2} \kappa A G}\left[\frac{r_{l} r_{u}+r_{l}+r_{u}}{4-r_{l} r_{u}}\right] \\
\lim _{\phi \rightarrow 0^{+}} \beta^{\prime}=\left[\frac{r_{l} r_{u}+r_{l}+r_{u}}{4-r_{l} r_{u}}\right]
\end{gathered}
$$

Note also that if both ends of the member are pin connections $\left(r_{l}=r_{u}=0\right)$ then $\zeta^{\prime}=0$ and $\beta^{\prime}$ in Eq. (16a) converges to Eq. (4), which means that lateral stiffness of a lean-on column is unaffected by shear deformation. Finally, the values of the end fixity factors can be calculated in terms of Eqs. (1) but with $R_{i, j}$ in Eq. (2) replaced by Eq. (20). The corresponding derivation is shown in Appendix A.

\subsection{Limiting Axial Load}

Eq. (17) is applicable for columns in axial compression that do not exceed the limits for either yielding or the rotational buckling failure mode, shown in Eq. (20).

$$
0 \leq P_{i} \leq P_{u, i}=\frac{\pi^{2} E I}{(K L)^{2}} ; \quad K=\frac{\pi}{\phi_{u}}
$$

where $P_{u, i}$ is the rotational buckling load of column $i$ with accounting for shear deformation, and $K$ is the effective length of the column obtained by solving for $\phi_{u}$, which is the minimum positive value of the axial load factor $\phi$ that satisfies Eq. (21).

$$
0=9 r_{u} r_{l}\left(\omega^{2}\left(2-2 \cos \phi^{\prime}\right)-\phi^{\prime} \sin \phi^{\prime}\right)+a_{1}{ }^{\prime}\left(\omega^{2} \phi^{\prime} \sin \phi^{\prime}-\left(\phi^{\prime}\right)^{2} \cos \phi^{\prime}\right)+\left(1-r_{u}\right)\left(1-r_{l}\right)\left(\phi^{\prime}\right)^{3} \sin \phi^{\prime}
$$

Although the exact solution cannot be obtained in closed form, Eq. (21) can conveniently be solved using root-finding methods such as the Newton-Raphson method [27]. In employing the root-finding methods, an initial guess of $\phi_{u}=\pi / K_{a p p}$ is recommended, where $K_{a p p}$ is an approximation of the effective length factor of the column given in Eq. (22) [28]. This will bring the initial guess of $\phi$ to within $4 \%$ of the exact value before considering the effect of shear 
deformation [28], ensuring that the solution will more easily converge to the $\phi_{u}$ value corresponding to the fundamental buckling mode.

$$
K_{a p p}=\sqrt{\frac{\left(\pi^{2}+\left(6-\pi^{2}\right) r_{u}\right) \times\left(\pi^{2}+\left(6-\pi^{2}\right) r_{l}\right)}{\left(\pi^{2}+\left(12-\pi^{2}\right) r_{u}\right) \times\left(\pi^{2}+\left(12-\pi^{2}\right) r_{l}\right)}}
$$

An approximate value of $P_{u}$ with accounting for shear deformation can also be obtained by applying the reduction factor in Eq. (23) to the buckling load obtained for Euler-Bernoulli columns, $P_{u, E B}[26]$.

$$
\begin{gathered}
P_{u}=P_{u, E B}\left[\frac{1}{1+P_{u, E B} / K A G}\right] \\
P_{u, E B} \approx \frac{\pi^{2} E I}{\left(K_{a p p} L\right)^{2}}
\end{gathered}
$$

Other methods of calculating $P_{u}$ are proposed by [6,24,29]. Note that if inelastic buckling is considered then the tangent modulus theory [18] can be applied in lieu of more accurate analysis methods. In such a case, the value of $E$ can be conservatively reduced by the empirical $\tau$ factor proposed by Yura \& Helwig [18] in Eq. (24) when solving for the critical inelastic buckling load.

$$
\tau=\left\{\begin{array}{c}
E=E_{0} \tau \\
-7.39 \frac{P}{P_{y}} \log _{10}\left(\frac{P}{0.85 P_{y}}\right) ; \quad \frac{P}{P_{y}} \leq \frac{1}{3} \\
1 ; \quad \text { otherwise }
\end{array}\right.
$$

Where $E_{0}=200 \mathrm{GPa}$. Note that Eq. (24) is the basis of the approach used in the AISC manual [30] for inelastic buckling analysis. The inelastic buckling load is therefore estimated by substituting $P=P_{u}$ in Eqs. (24) and solving with Eqs. (20) to obtain Eqs. (25).

$$
\begin{gathered}
P_{u}=0.85 P_{y}\left(0.7322^{F_{y}} / F_{e}\right) \\
F_{e}=\frac{\pi^{2} E_{0}}{(K L / r)^{2}}=\left\{\phi_{u}(E)\right\}^{2} \frac{E_{0} I}{L^{2} A}
\end{gathered}
$$


Eqs. (25) is similar to that of the AISC manual [30], which includes further adjustments in consideration of residual stresses and column imperfections. In Eq. (25b), $\phi_{u}$ is a function of $E$ due to its dependency on $r_{u}$ and $r_{l}$. As such, $P_{u}$ can be obtained via iteration of Eqs. (24) and (25), and replaces the right-hand term in Eq. (20) if inelastic buckling is considered. Also, the general equation for $G$ with considering plastic deformations is given in Eq. (26) [31].

$$
G=\frac{E_{0}}{2(1+v)+3 e}
$$

where $e$ may be taken as zero according to the incremental theory of plasticity, or $E_{\mathfrak{S}} / E_{0}-1$ according to the deformation theory of plasticity [31], and $E_{s}$ is the secant modulus. Before buckling occurs, $e=0$ regardless of which theory is used, due to the assumptions of small transverse deformations and concentric columns in this study. During buckling, however, it is shown in Becque [32] that $e$ may be taken as Eq. (27).

$$
e=\frac{1}{2}\left(E_{0} / E-1\right)
$$

Eq. (27) is based on plastic flow theory and is an improvement upon the incremental theory in that it considers the relationship between shear strain and stress increments at the onset of buckling in the absence of column initial imperfections [32]. As Eq. (27) is only applicable during the onset of buckling, it may only be used to obtain critical loads.

\subsection{Lateral stiffness of a semi-rigidly connected storey frame}

Within the applicable range $P_{i}<P_{u, i}$, the overall lateral stiffness of the storey, $\Sigma S$, when considering the combined effects of the specified imperfections and gravity loading is expressed in Eq. (28).

$$
\Sigma S=\sum_{i=1}^{n+1} S_{i}=\sum_{i=1}^{n+1}\left[\frac{12 E_{c, i} I_{c, i}}{L_{c, i}{ }^{3}} \beta_{i}^{\prime}\left(\frac{1}{1+\zeta_{i}^{\prime}}\right)\right]
$$


The use of Eq. (28) assumes that the lateral deflection of every column in the frame is equal, as is appropriate if the beams of the frame are connected to slabs and consistent with the methodology of [5]. The frame is stable when $\Sigma S>0$, and becomes unstable when $\Sigma S=0$. Columns that have negative lateral stiffness, $S_{i}<0$, rely on other columns in the storey to maintain stability. Note that similar to [21], the lateral stiffness of diagonal bracing can also be added to the storey-based lateral stiffness $\Sigma S$.

\section{$4 \quad$ Finite Element Validation}

A finite element analysis (FEA) was conducted via ABAQUS [17] to verify the critical axial load of single semi-rigidly connected column. The W310x60 column is bending about its weak axis $\left(I=125.1 \times 10^{6} \mathrm{~mm}^{4}, A=7,394.6 \mathrm{~mm}^{2}\right)$. The lower end is pinned $\left(r_{l}=0\right)$, while the upper end has a rotational rigidity of $R_{u}=3.98 \times 10^{7} \mathrm{Nm}$. Its length is varied in a parametric study. The critical load of a single column will be the minimum of either the sway load, $P_{s w}$, obtained by setting the lateral stiffness in Eq. (17) to zero, or the value of $P_{u}$ from either Eq. (20) or Eq. (25), depending on whether a purely elastic or inelastic buckling analysis is considered, respectively. Quadratic Timoshenko beam elements (B22) were used to account for shear deformation, while cubic Euler-Bernoulli beam elements (B23), which neglect shear deformation, were used as a comparison. Note that the value of $\omega$ corresponding to the results of the critical loads in ABAQUS for the B22 elements were found to be based on the assumption of Haringx [23] via Eq. (15b). The sway buckling and rotational buckling loads of the single column obtained from FEA were exact to the results obtained using the proposed equations in Eqs. (17), (20) and (25). To investigate the effect of the differing assumptions on the shear function, the theoretical results of applying Engesser's [22] assumption via Eq. (15a) were also obtained. With empirically considering the effects of inelastic buckling in Eqs. (24) and (25), it can be shown that $P_{s w}<P_{u}$ 
$\leq 0.85 P_{y}$ for the single column regardless of length. For very stocky columns, the values of $P_{u}$ and $P_{s w}$ obtained by the empirical approach in Eqs. (24) and (25) asymptotically approach $85 \%$ of the yielding load, $0.85 P_{y}=2,200 \mathrm{kN}$, as the slenderness ratio approaches zero. The critical sway loads, obtained from FEA and from Eq. (17), are plotted against the slenderness ratio of the column in Fig. (4).

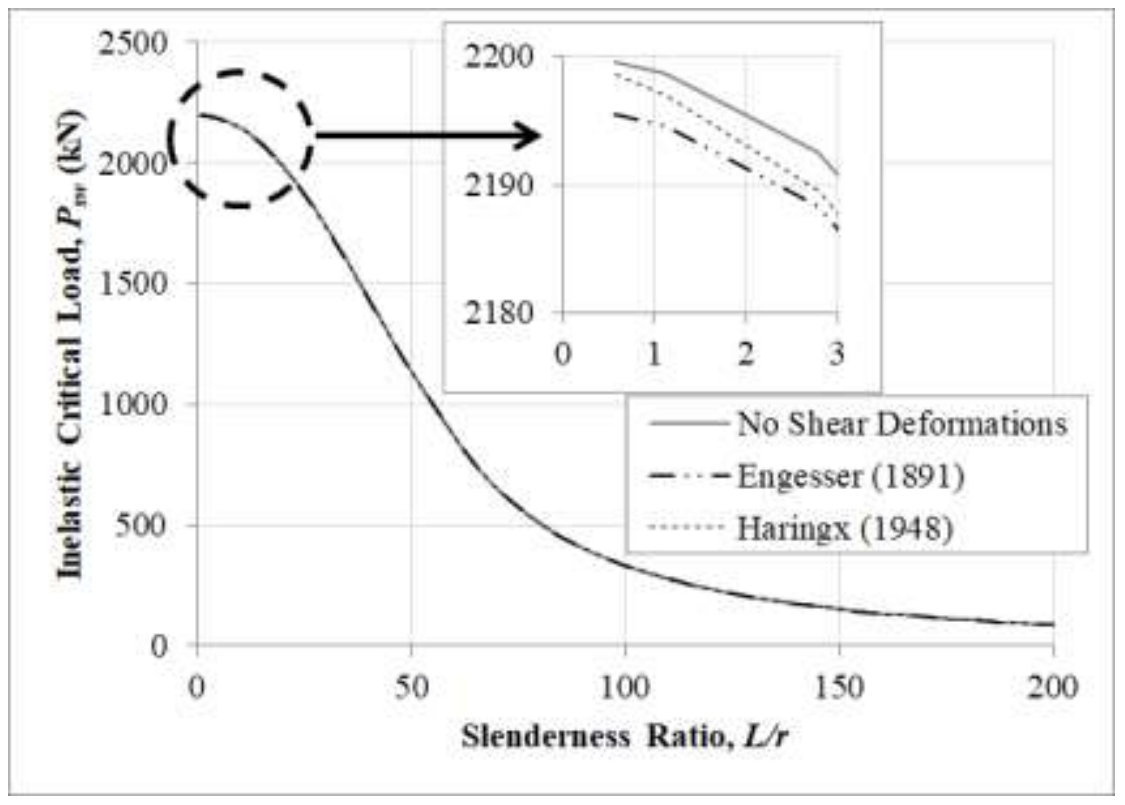

Figure 4 - Critical sway loads versus slenderness ratio

In Fig. (4), the obtained values of $P_{s w}$ with both the proposed method and FEA are identical when using Eq. (15b), as well as when shear deformation is neglected $(\eta=0)$. The theoretical value of $P_{s w}$ obtained from Engesser's assumption via Eq. (15a) is also plotted in Fig. (4). The difference between using the assumptions of Engesser [22] and Haringx [23] is within 0.003\% for $L / r>40$ and decreases with increasing slenderness ratio, but increases up to $0.2 \%$ as the slenderness ratio approaches zero. As such, accurate results can be obtained with using either assumption for the shear function for columns regardless of the slenderness ratio. For the single column, the difference in critical load with and without considering shear deformation is also relatively small (up to only $0.2 \%$ ) and can be neglected. For this single column, the effects of 
shear deformations on the critical load are negligible because the empirical tangent modulus, $\tau$, in Eq. (24) decreases very quickly with applied loading near the critical load, causing much larger decreases to the lateral stiffness over small increments of loading than that caused due to shear deformations alone. However, it will be demonstrated in the following sections that the shear deformations can have a significant influence on the results in other cases.

\section{$5 \quad$ Parametric Study}

A parametric study was conducted to demonstrate the effect of shear deformation on the reduction of critical loads in a lean-on frame system. Consider the lean-on frame system shown in Fig. (5), which consists of a supporting W460×97 column bending about its strong axis $\left(I_{x}=\right.$ $445 \times 10^{6} \mathrm{~mm}^{4}, A=12,300 \mathrm{~mm}^{2}$ ), connected to a series of $n-1$ lean-on columns. The base of the supporting column is rigidly connected to the ground $\left(r_{l}=1\right)$, while its upper end is pinned to the lean-on system $\left(r_{u}=0\right)$. The resulting effective length factor for the supporting column in the sway buckling mode is $K=0.7$. In order to assess the influence of the slenderness ratio, $L / r$, on the effect of shear deformations on the critical loads, the slenderness ratio of the supporting column is varied in this study from 5 to 100 by changing its length, $L_{1}$.

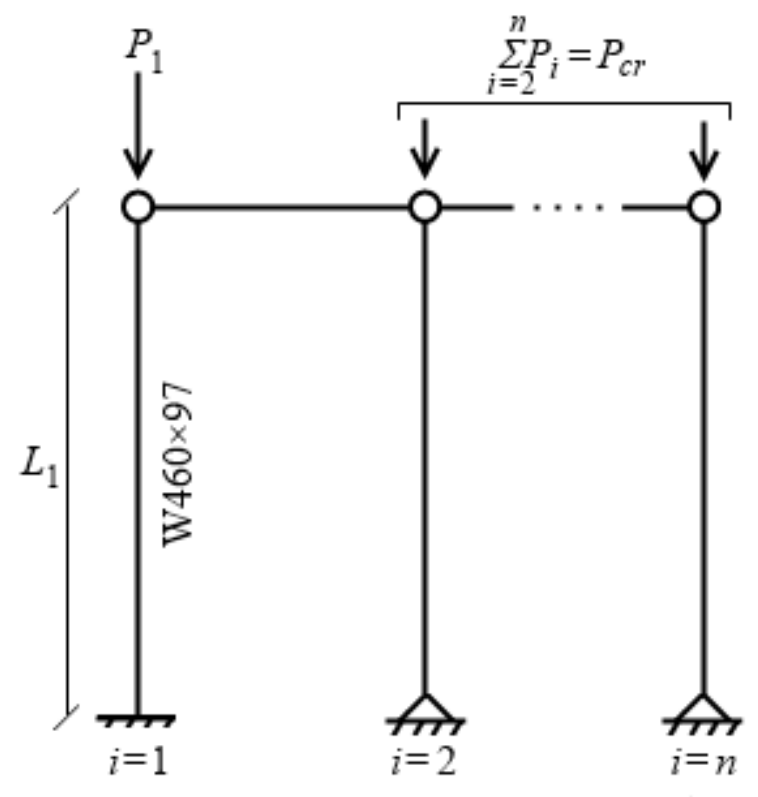




\section{Figure 5 - Portal frame geometry}

By definition, the ends of the lean-on columns have pinned connections $\left(r_{u}=r_{l}=0\right.$ and $\left.K=1\right)$. It can be shown that for lean-on columns, the lateral stiffness equation in Eq. (18) is simplified to Eq. (29).

$$
S_{i}=-P_{i} / L_{i}
$$

where $P_{i}$ is the compressive axial load the lean-on column and $L_{i}$ is the length of the lean-on column. Note that $S_{i}$ is independent of shear deformation. In the absence of axial loading $\left(P_{i}=0\right)$, a lean-on column has no contribution to the storey-based lateral stiffness. When a lean-on column is axially loaded, it relies on the supporting columns of a frame to maintain stability $\left(S_{i}<\right.$ $0)$. As such, no matter the lateral stiffness of the supporting column, its lateral stiffness can be diminished to zero via supporting a series of lean-on columns, as long as the axial loads in each lean-on column do not exceed their respective rotational buckling loads $\left(P_{i}<P_{u i}\right)$. The storeybased instability condition of the frame in Fig. (5) can be expressed by setting the storey-based lateral stiffness in Eq. (28) to zero, resulting in Eq. (30).

$$
S_{1}=\sum_{i=2}^{n} \frac{P_{i}}{L_{i}}, \quad P_{i}<P_{u, i}=\frac{\pi^{2} E_{i} I_{i}}{L_{i}^{2}} \forall i=2,3, \ldots, n
$$

where $S_{1}$ is the lateral stiffness of the supporting column. Assuming that the lengths of all of the lean-on columns are the same $\left(L_{i}=L\right)$ and that none of them rotationally buckle, the critical total load applied on the lean-on column system, $P_{c r}$ is defined via Eq.(31).

$$
P_{c r}=\sum_{i=2}^{n} P_{i}=L S_{i}
$$

Therefore, the critical total load of the frame is proportional to the lateral stiffness of the supporting column. It follows that a percentage reduction to the lateral stiffness of the supporting column will result in the same percentage reduction to the critical total load. Define $P_{s w}$ as the 
sway buckling load of the supporting column, which is the value of the axial load $P_{1}$ that diminishes the lateral stiffness of the supporting column to zero $\left(S_{1}=0\right)$ calculated with considering shear deformation. Thus, if $P_{1}=P_{s w}$ then $P_{c r}=0$ since the lean-on columns provide no contribution to the lateral stiffness even in the absence of loading. In this study, the value of $P_{1}$ is varied between zero (unloaded) and $P_{s w}$ (fully loaded) to assess the effect of increasing axial loads in the supporting column on $P_{c r}$.

The lateral stiffness $S_{1}$ was calculated with and without considering the effects of shear deformations via Eqs. (28) and (5), respectively. At first, where the effects of shear deformations were considered, both the Haringx [23] and Engesser [22] assumptions were compared via Eqs. (15b) and (15a), respectively. However, it was found that the difference in $P_{s w}$ obtained between using both assumptions was below $0.02 \%$ for $L / r=10$, and decreases for higher slenderness ratios (see Fig. 6).

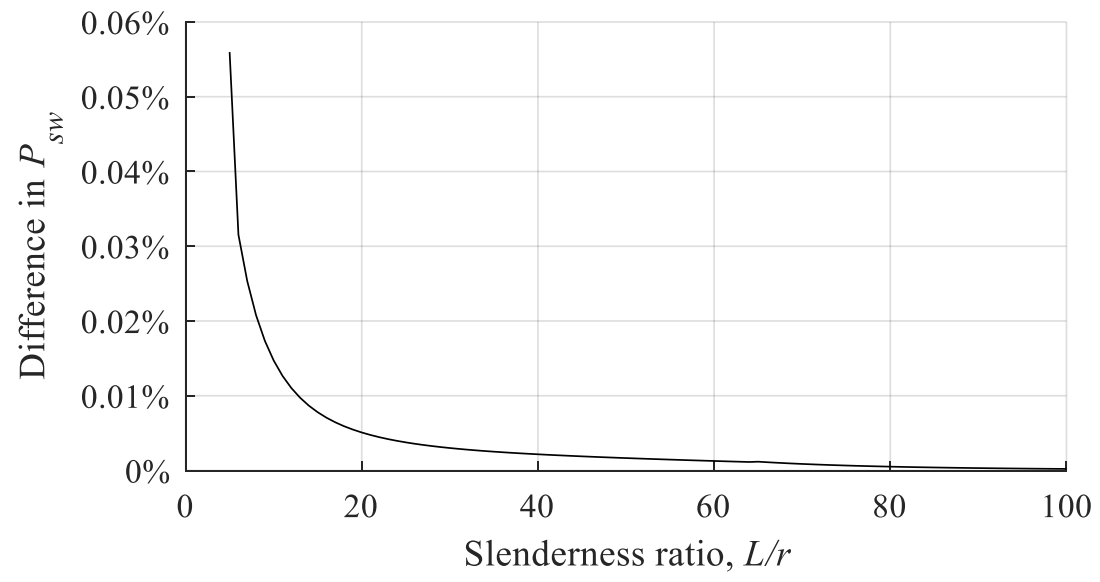

Figure 6 - Difference in $P_{s w}$ using the Haringx (1948) and Engesser (1891) assumptions Similar to the conclusion of the finite element validation, it was concluded that the use of either assumption produces virtually identical results. As such, only the results via using the Haringx [23] assumption via Eq. (15b) are reported where shear deformations are considered. A value of $\kappa=0.44$ for wide flange sections [25] is assumed. Assume that the yield stress of steel is 350 
$\mathrm{MPa}$ and the elastic modulus of steel is $200 \mathrm{GPa}$. The tangent modulus model in Eqs. (24) was used to account for the effects of partial yielding in the presence of high axial loads. The reduction in lateral stiffness of the supporting column due to considering shear deformations is plotted for varying slenderness ratios and load levels in Fig. (7).

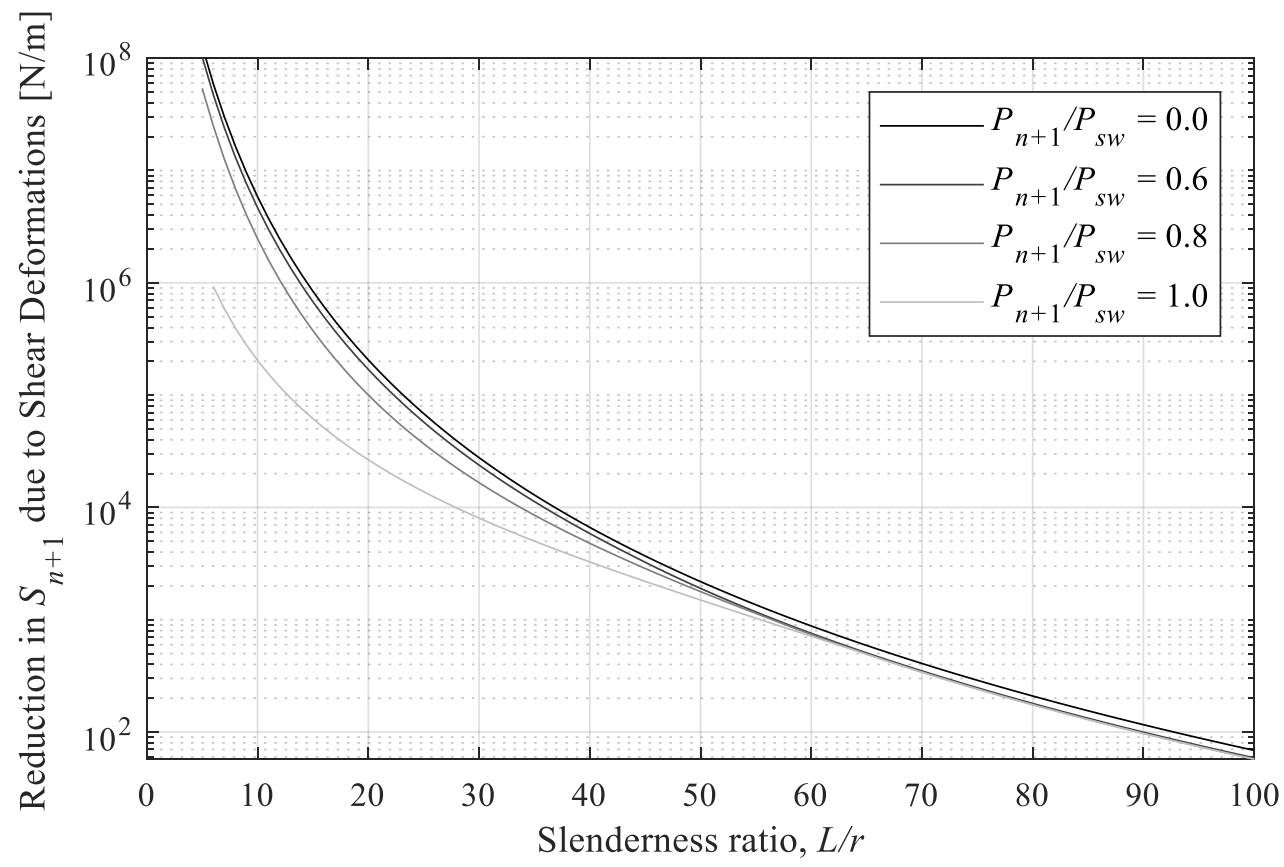

Figure 7 - Effect of shear deformations on $S_{1}$ with varying $L / r$ and loading levels

From Fig. (7), it can be seen that magnitude of the reduction in lateral stiffness due to accounting for shear deformation increases exponentially for low slenderness ratios. If the supporting column is unloaded $\left(P_{1}=0\right)$, then the difference can be as high as $1,000 \mathrm{kN} / \mathrm{m}$ for a slenderness ratio of 15 , but decreases to only $1.0 \mathrm{kN} / \mathrm{m}$ for a slenderness ratio of 60 . Above a slenderness ratio of 60 , the lateral stiffness is reduced by a negligible amount (below $1.0 \mathrm{kN} / \mathrm{m}$ ). The effect of increasing the axial load $P_{1}$ on the supporting column significantly decreases the reduction of lateral stiffness due to shear deformations at low slenderness ratios $(-93 \%$ for $L / r=15)$. However, note that as the magnitude of $P_{1}$ increases, the critical total load $P_{c r}$ will also be reduced. The percentage reduction to the critical total load of the frame, $P_{c r}$, as a result of considering shear 
deformations, is plotted in Fig. (8) for varying slenderness ratios and supporting column load levels. To be clear, a percentage reduction of $0 \%$ corresponds to no change in the calculated critical load between considering and neglecting the shear deformations via the Haringx [23] assumption, whereas a $100 \%$ reduction corresponds to a zero lateral stiffness when shear deformations are considered and an arbitrarily positive lateral stiffness when shear deformations are neglected.

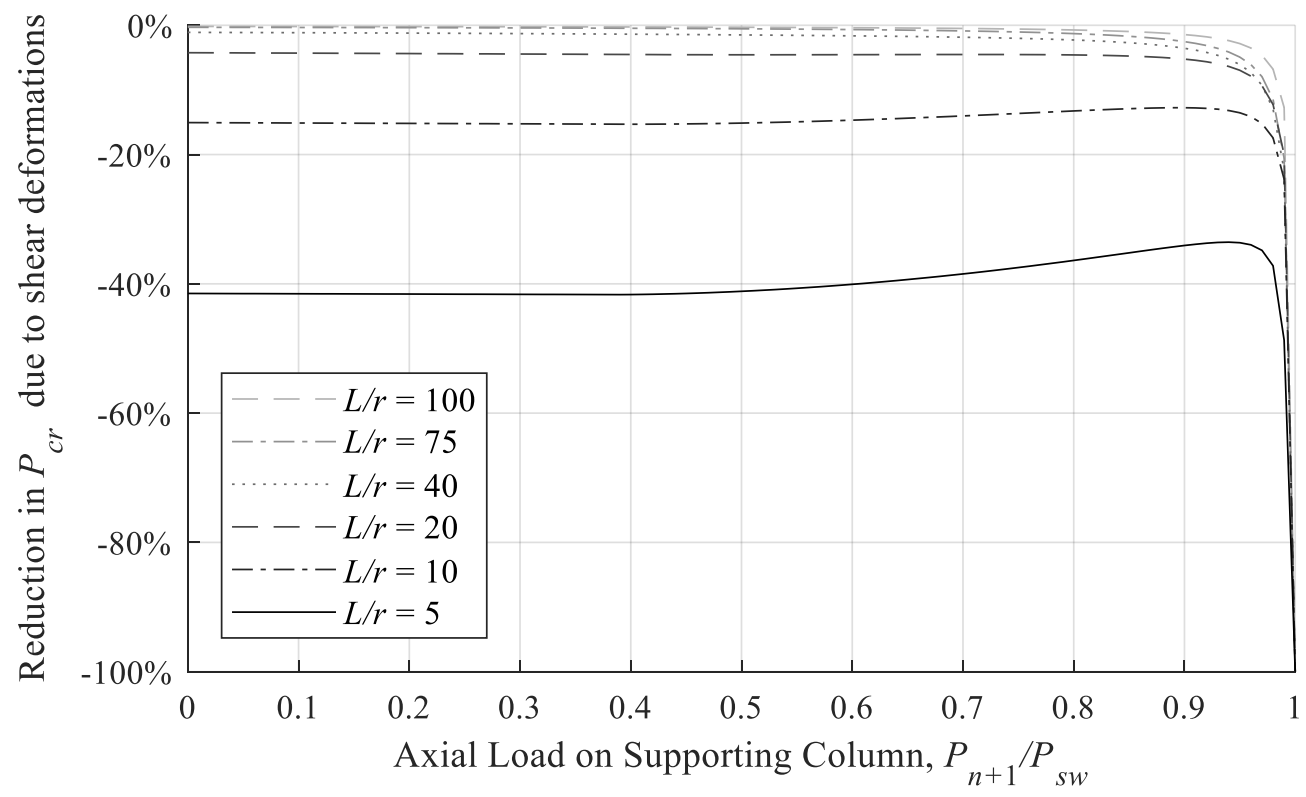

Figure 8 - Effect of shear deformations on $P_{c r}$ with varying $L / r$ and loading levels

In the absence of loading $\left(P_{1}=0\right)$, the reduction of $P_{c r}$ due to shear deformation can be significant for low slenderness ratios $(-41 \%$ for $L / r=5)$. For slenderness ratios below 20, the reduction is at least $4.2 \%$. The reduction is within $1.0 \%$ for $L / r=40$. Therefore, if a $1 \%$ reduction to the critical load is considered to be significant, it is recommended that shear deformations be considered for this frame if the supporting column has a slenderness ratio below $L / r=40$. By comparison, the threshold of significance with regards material reliability coefficients in standards is generally on the order of 0.05 , or 5\% [33]. However, the 
consideration for shear deformations always results in equal or lesser values of the lateral stiffness and critical loads in structural members compared to when they are neglected. As such, the threshold of significance for an error which is always non-conservative should typically be more stringent. A threshold of $1 \%$ has been adopted simply as a benchmark for the current study. Note that as $P_{1}$ increases, the percentage reduction of $P_{c r}$ due to shear deformation decreases slightly but increases to $100 \%$ at $P_{1}=P_{s w}$ for every curve. This is because at $P_{1}=P_{s w}$, the lateral stiffness of the supporting column is zero when considering shear deformation, so $P_{c r}=0$. However, if shear deformations are neglected then $P_{c r}$ will still be a positive value. It is not recommended in lean-on systems to load supporting columns near their sway capacities as that could result in the complete collapse of the frame. Based on the results of this analysis, shear deformations can have a significant influence on the critical sway loads in frames containing columns with low slenderness ratios. For this example, the effect of shear deformation on the critical load is significant (above 1.0\%) when the slenderness ratio of the supporting column is below 40, provided that rotational buckling does not occur elsewhere in the lean-on system. It is also shown that the lateral stiffness of a supporting column with slenderness ratio of 15 can be reduced by as much as $1,000 \mathrm{kN} / \mathrm{m}$. Although not recommended, if the supporting column is loaded near its sway load, the effects of shear deformation can reduce its lateral stiffness to zero and should definitely be considered, regardless of the slenderness ratio.

\section{$6 \quad$ Variable Loading}

To generalize the variable loading approach of $\mathrm{Xu}$ [5] to account for shear deformation, a modified minimization problem for determining the worst and best case distributions of axial loads resulting in the instability of an unbraced frame is formulated in Eqs. (32). 


$$
\min \text { or } \max : \sum_{i=1}^{n+1} P_{i}
$$

subject to:

$$
\begin{gathered}
\Sigma S=\sum_{i=1}^{n+1} S_{i}=\sum_{i=1}^{n+1}\left[\frac{12 E_{c, i} I_{c, i}}{L_{c, i}{ }^{3}} \beta_{i}^{\prime}\left(\frac{1}{1+\zeta_{i}^{\prime}}\right)\right]=0 \\
0 \leq P_{i} \leq P_{u, i}
\end{gathered}
$$

Eqs. (32) converges to Eqs. (7) when $\eta=0$, and can be solved using nonlinear constrained mathematical programming methods such as the GRG Nonlinear method [34] used in combination with Multi-Start [35]. From a design perspective, the results of the minimization problem can be used to identify the weakest columns of a frame, which can be fortified to increase the worst case loading capacity. Similarly, the results of the maximization problem indicate the relative proportions of loads that can be applied when assigning occupancies to the corresponding locations of the building in order to maximize the efficiency of the design. The results of Eqs. (32) are compared with the results of Eqs. (7) for the four-bay frame example from [5] illustrated in Fig. (9).

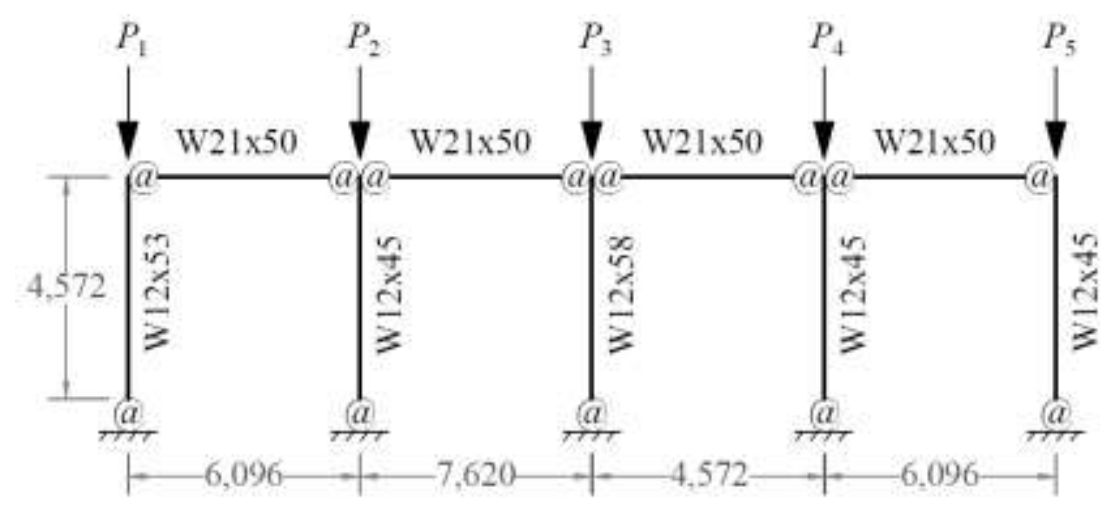

Figure 9 - Four-bay frame subjected to variable loading [5]

All of the connections are semi-rigid, with $r_{l}=0.2$ for all columns and $r_{N}=r_{F}=0.8$ for all beams, applicable when $E=E_{0}=200 \mathrm{GPa}$ in all members. Note that the end fixity factors will change if the tangent modulus theory is used. The columns and beams are numbered from left-toright. For the columns, $I_{c, 1}=177 \times 10^{6} \mathrm{~mm}^{4}, I_{c, 2}=I_{c, 4}=I_{c, 5}=145 \times 10^{6}$ and $I_{c, 3}=198 \times 10^{6} \mathrm{~mm}^{4}$. 
For all beams, $I_{b}=411 \times 10^{6} \mathrm{~mm}^{4}$. The areas of the columns are required when considering shear deformation, given as $A_{c, 1}=10,100 \mathrm{~mm}^{2}, A_{c, 2}=A_{c, 4}=A_{c, 5}=8,450 \mathrm{~mm}^{2}$ and $A_{c, 3}=11,000 \mathrm{~mm}^{2}$. The slenderness ratios of the columns range from 34 to 35 , indicating that the columns are within the inelastic range of slenderness. For wide-flange sections, $\kappa=0.44$ [25]. The yield stress is assumed to be $350 \mathrm{MPa}$ and Poisson's ratio is taken as 0.3 for all members. The rotational buckling loads for each column in the frame were calculated using Eqs. (25) and are shown in Table 1.

Table 1 - Rotational buckling loads of columns for numerical example

\begin{tabular}{cccccc}
\hline & $P_{u, 1}$ & $P_{u, 2}$ & $P_{u, 3}$ & $P_{u, 4}$ & $P_{u, 5}$ \\
\hline Without shear deformation & $2,942 \mathrm{kN}$ & $2,461 \mathrm{kN}$ & $3,206 \mathrm{kN}$ & $2,461 \mathrm{kN}$ & $2,460 \mathrm{kN}$ \\
With shear deformation & $2,948 \mathrm{kN}$ & $2,466 \mathrm{kN}$ & $3,213 \mathrm{kN}$ & $2,466 \mathrm{kN}$ & $2,466 \mathrm{kN}$ \\
\hline
\end{tabular}

Based on Table 1, the rotational buckling loads of the columns differ by less than $0.25 \%$ between neglecting and considering shear deformations. The reason for such a small difference is that $P_{u}$ is not affected by $\zeta$ 'when solving Eq. (21). The effect of shear deformation is accounted for via $\eta$, which appears in only the equations for $\omega$ and $\zeta^{\prime}$. As such, a solution that is independent of $\zeta^{\prime}$ such as the value of $P_{u}$ will not be as significantly influenced by shear deformation. Moreover, when $P=P_{u}$, the tangent modulus reduction factor in Eq. (24) is low ( $\tau \approx 0.05$ for each column), reducing the value of $\eta$ significantly and thus further reducing the influence of shear deformations on the rotational buckling load. Note that the results throughout this example obtained with considering shear deformations were once again virtually identical using either of the Engesser [22] or Haringx [23] assumptions.

\subsection{Worst Case Variable Loading}

The worst case gravity loading scenario is obtained by minimizing Eq. (32a), and the results for the numerical example are shown in Table 2. 
Table 2 - Worst case scenario of axial loading for numerical example

\begin{tabular}{|c|c|c|c|c|c|c|}
\hline & $P_{1}$ & $P_{2}$ & $P_{3}$ & $P_{4}$ & $P_{5}$ & Total \\
\hline Without shear deformation & $0.0 \mathrm{kN}$ & $0.0 \mathrm{kN}$ & $0.0 \mathrm{kN}$ & $0.0 \mathrm{kN}$ & $2,466 \mathrm{kN}$ & $2,466 \mathrm{kN}$ \\
\hline With shear deformation & $0.0 \mathrm{kN}$ & $0.0 \mathrm{kN}$ & $0.0 \mathrm{kN}$ & $0.0 \mathrm{kN}$ & $2,460 \mathrm{kN}$ & $2,460 \mathrm{kN}$ \\
\hline
\end{tabular}

Rotational buckling of Column 5 governs the worst case scenario for this example. Note that Column 5 has a marginally lower rotational buckling load than those of Columns 4 and 2 due to the varying lengths of the connection beams. In fact, contrary to what is suggested in [5], it can be shown that the minimum solution to Eqs. (5) and (32) always consists of loading in only one column. The reason for this is that in [5], the effective length factor is approximated via Eq. (22), and the resulting values of $P_{u}$ are not exact. However, the accurate determination of $P_{u}$ is necessary because as $P_{i}$ approaches $P_{u, i}$, the lateral stiffness of the column decreases asymptotically towards negative infinity [5]. Thus, given the assumption that the entire frame deflects laterally as a unit, instability of the frame will occur as Eq. (28) diminishes to zero for some value of $P_{i} \leq P_{u, i}$. If $P_{u}$ is underestimated, the domain of the minimization problem will be truncated and in a way that eliminates the consideration of potentially infinite reduction to the frame lateral stiffness as $P_{i}$ approaches $P_{u, i}$. Note that it is shown in [36] that for column with high end fixity factors the slope of the curve of lateral stiffness versus $P_{i}$ gradually becomes steeper for $P_{i}$ approaching $P_{u, i}$, as shown via the dashed line in Fig. (10).

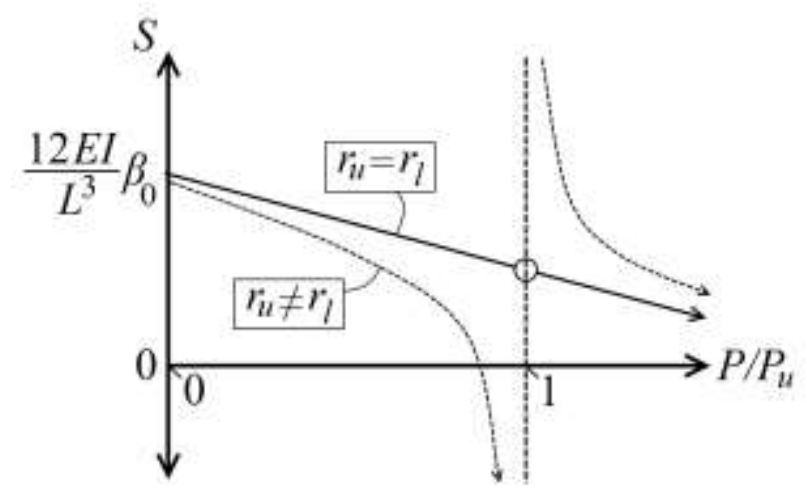




\section{Figure 10 - Curves of lateral stiffness versus axial load for varying end fixities}

However, as the end fixity factors of a column decrease, the curve of lateral stiffness versus $P_{i}$ decreases more sharply towards negative infinity for $P_{i}$ approaching $P_{u, i}$. In fact, for the special case of a lean-on column $\left(r_{l}=r_{u}=0\right)$, the curve becomes so steep that the lateral stiffness abruptly becomes discontinuous at $P_{i}=P_{u, i}$, corresponding to rotational buckling and shown via the solid line in Fig. (7). Based on the above rationale, the solution to the minimization problems in Eqs. (5) and (32) must satisfy Eq. (33).

$$
\sum_{i=1}^{n+1} P_{i} \leq \min _{i \in[1, n+1]}\left\{P_{u, i}\right\}
$$

In many cases, the minimum case will consist of a single column being loaded until $P_{i} \approx P_{u, i}$. However, the instability will may take the form of a local failure at the location of the column which is loaded near its rotational buckling load, rather than failure of the entire storey. If load redistribution measures are in place, a progressive collapse analysis can be conducted to determine if the rest of the frame can maintain stability if the buckled column is deleted from the analysis and its load is redistributed to other columns. Such an analysis would be out of the scope of this paper, which focuses mainly on the effect of shear deformation towards the lateral stability of unbraced frames. Regardless of whether or not local failure will occur when a column approaches its rotational buckling load, instability will still occur to some degree, and can be detected via the minimization problem.

To assess whether or not shear deformations will influence the minimum case loading solution when rotational buckling is not imminent, the slenderness ratios of the columns in the frame were increased until rotational buckling no longer governed the minimum solution result (i.e. $P_{i}$ $\leq 0.95 P_{u, i}$ for all $i$ in the minimum case). It was found that a length of $L=12.8 \mathrm{~m}$ for all the columns was required in order for this to occur, corresponding to slenderness ratios between 90 
and 94. Since the lengths of the columns were changed proportionally, Column 5 remained the weakest and only column to be loaded in the minimum case solution $\left(P_{1}=P_{2}=P_{3}=P_{4}=0\right)$. The rotational buckling load of Column 5 was $P_{u, 5}=2004.6 \mathrm{kN}$ and $2010.7 \mathrm{kN}$ with and without considering shear deformation, respectively. In the minimum solution, instability of the frame occurred when $P_{5}=1988.7 \mathrm{kN}$ in the minimum scenario with neglecting shear deformations. When considering shear deformations, the minimum case was $P_{5}=1981.6 \mathrm{kN}$, corresponding to only a $0.4 \%$ decrease in the total load. As demonstrated in the previous section, if the slenderness ratio is further increased, the effect of shear deformation on the result will only diminish. As such, it is shown that for this example the minimum case solution is not significantly affected by shear deformation.

Between neglecting and considering shear deformations, the worst case scenario loading was affected by up to $0.4 \%$ in this example. The reason for this negligible difference is due to the fact that the rotational buckling load is not significantly affected by the effect of shear deformation in these columns, but governs the worst case instability failure of the original frame. The slenderness ratio of the columns was increased to avoid rotational buckling, but it was found that when rotational buckling no longer governed, the slenderness ratio became too high for shear deformations to have a significant influence on the results.

\subsection{Best Case Variable Loading}

The best case scenario is obtained by maximizing Eq. (32a) for the original frame in Fig. (9), and the results are shown in Table 3.

Table 3 - Best case scenario of axial loading for numerical example

\begin{tabular}{ccccccc}
\hline & $P_{1}$ & $P_{2}$ & $P_{3}$ & $P_{4}$ & $P_{5}$ & Total \\
\hline Without shear deformation & $2,702 \mathrm{kN}$ & $2,225 \mathrm{kN}$ & $2,877 \mathrm{kN}$ & $2,217 \mathrm{kN}$ & $2,253 \mathrm{kN}$ & $\mathbf{1 2 , 2 7 4} \mathbf{~ k N}$ \\
With shear deformation & $2,692 \mathrm{kN}$ & $2,219 \mathrm{kN}$ & $2,866 \mathrm{kN}$ & $2,211 \mathrm{kN}$ & $2,247 \mathrm{kN}$ & $\mathbf{1 2 , 2 3 3} \mathbf{~ k N}$ \\
\hline
\end{tabular}


Between neglecting and considering shear deformations, the best case total load was affected by only $0.3 \%$ in this example. The effect of shear deformations has a negligible influence on the best case solution due to the fact that in the loading scenarios tabulated in Table 2, the columns are all loaded within $89 \%$ to $92 \%$ of their corresponding rotational buckling loads, $P_{u}$. As discussed previously, the lateral stiffness of a column begins to decrease very sharply as the axial loads approach $P_{u}$. In this case, since rotational buckling is imminent, a difference of only $39 \mathrm{kN}$ to the total load of the frame is enough to account for the decrease in lateral stiffness that results from considering the effect of shear deformation while maintaining the instability condition.

Upon further investigation, it was found that if the three interior columns were changed to leanon columns $\left(r_{l}=r_{u}=0\right)$ and the same maximization problem was solved for the modified frame, the effect of shear deformations would more significantly affect the best case loading scenario, shown by the best case solutions in Table 4 .

Table 4 - Best case scenario of axial loading for modified numerical example

\begin{tabular}{ccccccc}
\hline & $P_{1}$ & $P_{2}$ & $P_{3}$ & $P_{4}$ & $P_{5}$ & Total \\
\hline Without shear deformation & $1,037 \mathrm{kN}$ & $2,069 \mathrm{kN}$ & $2,668 \mathrm{kN}$ & $2,141 \mathrm{kN}$ & $815 \mathrm{kN}$ & $\mathbf{8 , 7 3 0 ~ k N}$ \\
With shear deformation & $1,144 \mathrm{kN}$ & $2,244 \mathrm{kN}$ & $2,761 \mathrm{kN}$ & $2262 \mathrm{kN}$ & $219 \mathrm{kN}$ & $\mathbf{8 , 6 3 1 ~ k N}$ \\
\hline
\end{tabular}

Note that the rotational buckling loads of the modified columns are $P_{u, 2}=2,350 \mathrm{kN}, P_{u, 3}=3,069$ $\mathrm{kN}$, and $P_{u, 4}=2,350 \mathrm{kN}$ without accounting for shear deformation, respectively. With considering shear deformation, the rotational buckling loads are reduced slightly to $P_{u, 2}=2,345$ $\mathrm{kN}, P_{u, 3}=3,062 \mathrm{kN}$, and $P_{u, 4}=2,345 \mathrm{kN}$, respectively. Also, the best case solutions presented in Table 4 are not unique as the interior column loads can be re-distributed between the interior columns without changing the total lateral stiffness of the frame, based on Eq. (29), as long as the loads are below their respective rotational buckling loads. The difference between the best 
case loads with and without considering shear deformation in the modified example is $1.2 \%$, which is within the threshold of significance defined in Section 5. Note that the slenderness ratios of the exterior columns in this example are between 34 and 35, which is within the range of significance in shear deformation reported in the previous section. As such, shear deformations have the potential to significantly influence the best case loading scenario where supporting columns have low slenderness ratios and are not loaded close to their rotational buckling limits.

\section{Conclusion}

This study has demonstrated that in certain cases, shear deformations can significantly reduce the buckling loads of unbraced steel frames, and should not always be neglected in storey-based stability analyses. Moreover, for the compressively loaded columns in this study, the effect of shear deformation always reduces the lateral stiffness, and consequently, the critical loads of a frame subjected to gravity loading. The lateral stiffness equation for a semi-rigidly connected column with considering shear deformation was derived using the Timoshenko beam assumption. The consideration of shear deformation can conveniently be accomplished by employing the shear flexibility coefficient [6], $\eta$, in the lateral stiffness equation. With assuming that all of the columns in an unbraced frame experience equal lateral displacements, the lateral stiffness equation of an unbraced storey frame is also presented. Instability of the frame occurs when its lateral stiffness diminishes to zero as a result of axial loads. It is noted that the lateral stiffness equations proposed by $\mathrm{Xu}$ [5] are a special case of the proposed equations where $\omega=1$. The proposed method was validated using finite element analysis, and the differences between the critical loads with and without considering shear deformation were found to be significant where columns have low slenderness ratios and are not loaded close to their rotational buckling limits. 
Particularly, based on the numerical examples it is recommended that the critical loads of leanon frames with supporting columns with slenderness ratios below 40 will be significantly influenced by shear deformation. The effect of using competing assumptions of the shearing angle proposed in the literature by Engesser [22] and Haringx [23] are also compared and were found to produce virtually identical results for the structural applications covered within the scope of this study. The variable loading approach first proposed by $\mathrm{Xu}$ [5] to determine the worst and best case distributions of axial loads causing instability of a frame was also generalized to account for shear deformation, and demonstrated via numerical example. Based on the results of the example, the rotational buckling is not as heavily influenced by shear deformations. It was also shown that the total axial load in the worst case solution to the minimization problem in the variable loading approach cannot exceed the minimum rotational buckling load of the columns in a frame. The effect of shear deformation was not found to be significant for the minimum loading case since either rotational buckling will govern the solution or the columns will become too slender. In contrast, the best case loading scenario was found to be significantly influenced by shear deformations (a difference of $1.0 \%$ in the example). Although the effects of shear deformation may be neglected in stability analysis when all of the columns are sufficiently slender or where rotational buckling governs the failure of the frame, there are cases when the critical loads in frames containing low-slenderness columns are significantly affected by shear deformations via the proposed method which need to be considered.

\section{Acknowledgements}

The authors wish to thank the National Science and Engineering Research Council (NSERC) (RGPIN-203154-2013) of Canada for the financial support of this work. 


\section{References}

1. Yura, J.A. (1971). The effective length of columns in unbraced frames. Engineering Journal, vol. 8, no. 2, pp 37-42.

2. LeMessurier, W. (1977). A practical method of second order analysis Part 2 - Rigid Frames. Engineering Journal, vol. 14, no. 2, pp. 49-67.

3. Lui, E.M. (1992). A novel approach for K factor determination. Engineering Journal, vol. 29, pp. 150-159.

4. Aristizabal-Ochoa, JD. (1997). Storey stability of braced, partially braced, and unbraced frames: classical approach. Journal of Structural Engineering, vol. 123, no. 6, pp. 799-807.

5. Xu, L. (2001). The buckling loads of unbraced PR frames under non-proportional loading. Journal of Constructional Steel Research, vol. 58, pp. 443-465.

6. Wang, C-M., Xiang, Y., Kitipornchai, S. (1991). Buckling of restrained columns with shear deformation and axial shortening. Journal of Engineering Mechanics, vol. 117, no. 9, pp. 1973-1989.

7. Lin, F.J., Glauser, E.C. and Johnston, B.G. (1970). Behavior of laced and battened structural members. Journal of the Structural Division, vol. 96, no. 7, pp. 1377-1401.

8. Ziemian, R. (2010). Guide to Stability Design Criteria for Metal Structures. Wiley and Sons Ltd., $6^{\text {th }}$ edition.

9. Truesdell, C. and Euler, L. (1960). The rational mechanic of flexible or elastic bodies 1638-1788. Venditioni Exponunt Orell Fussli Turici.

10. McGuire, W., Gallagher, R.H, Ziemian, R.D. (2000). Matrix Structural Analysis. John Wiley \& Sons Inc, $2^{\text {nd }}$ edition. 
11. Timoshenko, S.P. (1916). "Course of Elasticity Theory. Part 2 - Columns and Plates". A.E. Collins Publishers, St. Petersburg.

12. Méndez-Sánchez, R.A ., Morales, A. and Flores, J. (2005). Experimental check on the accuracy of Timoshenko's beam theory. Journal of Sound and Vibration, vol. 279, no. 1, pp. 508-512.

13. Díaz-de-Anda, A., Flores, J., Gutiérrez, L., Méndez-Sánchez, R.A., Monsivais, G. and Morales, A. (2012). Experimental study of the Timoshenko beam theory predictions. Journal of Sound and Vibration, vol. 331, no. 26, pp. 5732-5744.

14. Thomas, D.L., Wilson, J.M. and Wilson, R.R. (1973). Timoshenko beam finite elements. Journal of Sound and Vibration, vol. 31, no. 3, pp. 315-330.

15. Dawe, D.J. (1978). A finite element for the vibration analysis of Timoshenko beams. Journal of Sound and Vibration, vol. 60, no. 1, pp. 11-20.

16. Friedman, Z. and Kosmatka, J.B. (1993). An improved two-node Timoshenko beam finite element. Computers \& Structures, vol. 47, no. 3, pp. 473-481.

17. Simulia, (2012). ABAQUS/CAE User's Manual (version 6.12). Dassault Systèmes Simulia Corp., Providence, RI.

18. Yura, J. A., Helwig, T.A. (2005). Bracing for stability. AISC and SSRC.

19. Monforton, G., Wu, T. (1963). Matrix analysis of semi-rigidly connected frames. Jouranl of the Structural Division, vol. 89, no 6, pp. 13-42.

20. Xu, L., Liu, Y. (2002). Storey-based effective length factors for unbraced PR frames. Engineering Journal, vol. 39, no. 1, pp. 13-29.

21. Xu, L., Liu, Y. (2002). Storey stability of semi-braced frames. Journal of Constructional Steel Research, vol. 58, pp. 467-491. 
22. Engesser, F. (1891). Die knickfestigkeit gerader stabe. Zentralbl, Bauverwaltung, vol. 11, pp. $483-486$.

23. Haringx, J.A. (1948). On highly compressible helical springs and rubber rods, and their application for vibration-free mountings, I-III (Reports R 94, 101, 109). Philips research reports, Eindhoven, pp. 1-97.

24. Ziegler, H. (1982). Arguments for and against Engesser's buckling formulas. IngenieurArchiv, vol. 52, no. 1982, pp. 105-113.

25. Cowper, G.R. (1966). The shear coefficient in Timoshenko's beam theory. Journal of Applied Mechanics, vol. 33, no. 2, pp. 335-340.

26. Timoshenko, S.P. and Gere, J.M. (1961). Theory of elastic stability, McGraw-Hill, New York, N.Y.

27. Ypma, T. J. (1995). Historical development of the Newton-Raphson method. SIAM Review, vol. 37 , no. 4 , pp. 531-551.

28. Xu, L. (2003). A NLP approach for evaluating storey-buckling strengths of steel frames under variable loading. Structural and Multidisciplinary Optimization, vol. 25, no. 2, pp. $141-150$.

29. Banerjee, J.R. and Williams, F.W. (1994). The effect of shear deformation on the critical buckling of columns. Journal of Sound and Vibration, vol. 174, no. 5, pp. 607-616.

30. AISC. (2011). Steel Construction Manual, $13^{\text {th }}$ ed. Chicago, IL.

31. Lau, S.C.W. and Hancock, G.J. (1989). Inelastic buckling analyses of beams, columns and plates using the spline finite strip method. Thin-Walled Structures, vol. 7, no. 1989, pp. 213-238. 
32. Becque, J. (2016). The application of plastic flow theory to inelastic column buckling. International Journal of Mechanical Sciences, vol. 111, pp. 116-124.

33. CSA. (2014). Design of Steel Structures (CSA S16). CSA Group, Toronto, ON.

34. Lasdon, L.S., Fox, R.L, Ratner, M.W. (1973). Nonlinear optimization using the generalized reduced gradient method. Case Western Reserve University, Cleveland, $\mathrm{OH}$.

35. György, A. and Kocsis, L. (2011). Efficient multi-start strategies for local search algorithms. Journal of Artificial Intelligence Research, vol. 41, no. 2011, pp. 407-444.

36. Zhuang, Y. (2013). Storey-based stability analysis of unbraced steel frames at ambient and elevated temperatures (Ph.D. thesis). University of Waterloo, Waterloo, ON. 


\section{Appendix A - Rotational Stiffness Contribution of a Timoshenko Member}

Shear deformations not only affect the lateral stiffness of columns directly, but also the effective rotational stiffness of beams connected at the ends of columns $\left(R_{i, j}\right)$, and thus subsequently also the end fixity factors of the columns. A more generalized form of Eq. (2) for calculating the effective rotational stiffness provided by a connecting beam with accounting for shear deformations in the connected beams is derived in this appendix. Consider first the member with ends $A$ and $B$ in Fig. (A1).

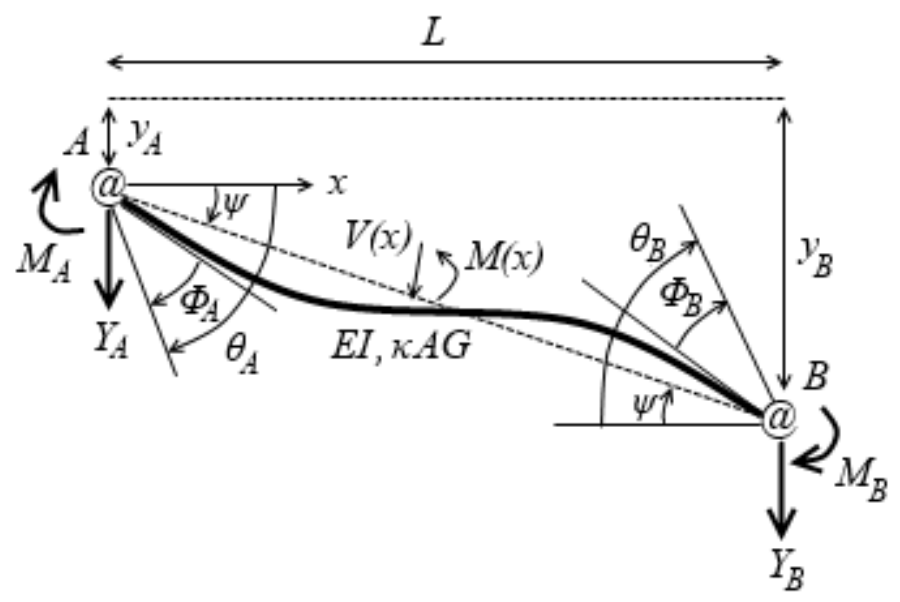

\section{Figure A1 - Deformation of a typical semi-rigidly connected member}

where $\psi$ is the chord rotation, $\theta$ is the connection rotation with respect to the original orientation, and $\Phi$ is the relative rotation between the end of the member and the rotation of the connection. $y(x)$ is the transverse displacement, or deflection, of the member. The transverse reaction $Y_{A}$ and $Y_{B}$ are also present at the ends. The connections on either end of the member have rotational stiffness $Z_{A}$ and $Z_{B}$, and the corresponding end fixity factors, $r_{A}$ and $r_{B}$, can be expressed via Eq. (A1).

$$
r_{A}=\frac{1}{1+3 E I /\left(Z_{A} L\right)} ; \quad r_{B}=\frac{1}{1+3 E I /\left(Z_{B} L\right)}
$$


As such, the end moments $M_{A}$ and $M_{B}$ are expressed via Eq. (A2) as functions of the relative rotations between the connections and their respective member ends, $\Phi$, in the positive clockwise direction.

$$
M_{A}=\Phi_{A} Z_{A} ; \quad M_{B}=\Phi_{B} Z_{B}
$$

The equation of external moment equilibrium for Fig. (A1) is expressed in Eq. (A3).

$$
\Phi_{A} Z_{A}+\Phi_{B} Z_{B}+Y_{B} L=0
$$

Likewise, the expressions of internal moment and shear associated with the Timoshenko [11] beam theory are given in Eqs. (A4).

$$
\begin{gathered}
M(x)=-E I \frac{d \varphi}{d x}=\Phi_{A} Z_{A}+Y_{B} x \\
V(x)=\kappa A G\left(-\varphi+\frac{d y}{d x}\right)=Y_{B}
\end{gathered}
$$

The system of two differential equations in Eqs. (4) is then solved for the deflected shape, $y(x)$, and the shear angle normal to the cross-section of the member, $\varphi(x)$. The solution is given in Eqs. (A5).

$$
\begin{gathered}
y(x)=C_{1}+C_{2} x+\frac{Y_{B}}{\kappa A G} x-\frac{Z_{A} \Phi_{A}}{2 E I} x^{2}-\frac{Y_{B}}{6 E I} x^{3} \\
\varphi(x)=C_{2}-\frac{Z_{A} \Phi_{A}}{E I} x-\frac{Y_{B}}{2 E I} x^{2}
\end{gathered}
$$

As part of the process required to solve for the integration constants $C_{1}$ and $C_{2}$, the four boundary conditions listed in Eqs. (A6) are substituted into Eqs. (A5).

$$
\begin{gathered}
y(0)=y_{A} \\
y(L)=y_{B} \\
\varphi(0)=\theta_{A}-\Phi_{A} \\
\varphi(L)=\theta_{B}-\Phi_{B}
\end{gathered}
$$


The system of five equations comprising of Eqs. (A6) and (A3) can be linearly solved for the unknowns $C_{1}, C_{2}, Y_{B}, \Phi_{A}$ and $\Phi_{B}$ as functions of all other variables. Substituting the results for $\Phi_{A}$ and $\Phi_{B}$ in Eq. (A2) results in Eq. (A7).

$$
\left[\begin{array}{c}
M_{A} \\
M_{B}
\end{array}\right]=\left[\begin{array}{cc}
Z_{A} & 0 \\
0 & Z_{B}
\end{array}\right]\left[\begin{array}{l}
\Phi_{A} \\
\Phi_{B}
\end{array}\right]=\mathbf{C}_{\mathbf{2 \times 4}}\left[\begin{array}{c}
\theta_{A} \\
\theta_{B} \\
y_{A} \\
y_{B}
\end{array}\right]
$$

where $C_{2 \times 4}$ is a rotational stiffness coefficient matrix. Let $A$ be the near end of the member to the joint for which the end fixity factor of column $i$ is being calculated. Then rearranging Eq. (A7) and applying Eq. (A1) yields the following result for $R_{i, j}=M_{A} / \theta_{A}$, which is the equivalent rotational stiffness provided to the connecting member at end $A$.

$$
R_{i, j}=\frac{6 E_{b, j} I_{b, j} r_{N, j}}{L_{b, j}}\left[\frac{2+6 \eta_{j} r_{F, j}+r_{F, j}\left(1-6 \eta_{j}\right) v_{j}-\left(2+r_{F, j}\right) w_{j}}{4-r_{N, j} r_{F, j}+12 \eta_{j}\left(r_{N, j}+r_{F, j}+r_{N, j} r_{F, j}\right)}\right]
$$

where $\eta$ is the shear flexibility coefficient of beam $j$ from Eq. (6) and $w$ is given in Eq. (A9).

$$
w=\frac{y_{B}-y_{A}}{\theta_{A} L}
$$

$w$ is related to the differential column axial displacement during buckling since $y_{B}$ and $y_{A}$ are the end displacements of the beam. $w=0$ is assumed to be zero as consistent with the assumption of [20] in deriving Eq. (2). Eq. (A8) replaces Eq. (2) when considering shear deformations, but converges to Eq. (2) when shear deformations are neglected (i.e. when $\eta=0$ ). 